\title{
Ising Model Phase Transition Calculation for Ferro-Paramagnetic Lattice
}

\author{
Dhia`a K. Khudier ${ }^{1}$, Nabeil I. Fawaz ${ }^{2, *}$ \\ ${ }^{1}$ College of Pharmacy, University of Anbar, P.O. Box 55, Al-Anbar, Iraq \\ ${ }^{2}$ College of Science, University of Anbar, P.O. Box 55, Al-Anbar, Iraq \\ *E-mail address: nifawaz@yahoo.com
}

\begin{abstract}
The position of the phase transition in the two dimensional Ising model were determined by using Monte Carlo simulation in a quadratic for area of variable length with external magnetic field switched off $(B=0)$. The magnetization $(M)$ per site $(\mu)$, magnetic susceptibility $(\chi)$ of a ferromagnetic and paramagnetic materials were calculated as a function of temperature $T$ for $(20 \times 20,40 \times 40,60 \times 60),(80 \times 80,120 \times 120,200 \times 200)$ spin lattice interactions. Nearest neighbor interaction is assumed (i.e. each spin has 4 neighbors); uses periodic boundary conditions. The Curie temperature $\left(T_{C}=2.27 \mathrm{~J} / k_{B}\right)$ is determined by measuring the magnetic susceptibility at which the ferromagnetic and paramagnetic undergoes a phase change from order to disorder. There is thus a phase transition defined by the Curie temperature. The Monte Carlo method were used to check these results and to confirm the phase transition. The data are analyzed using the Curie-Weiss law which contains the Curie temperature as a parameter.
\end{abstract}

Keywords: Monte Carlo simulation; magnetic; Ferromagnetic materials

\section{INTRODUCTION}

Ferromagnetic materials show ferromagnetic behavior only below a critical temperature called the Curie temperature, above which the material has normal paramagnetic behavior.

The approach to ferromagnetism as a function of temperature from above is described by the Curie-Weiss Law which gives the magnetic susceptibility as a function of temperature.

$$
\chi=\mu-1=\frac{C}{T-T_{C}}
$$

where $\chi$ and $\mu$ are the magnetic susceptibility and relative magnetic permeability of the material respectively. $C$ is a constant characteristic for a given substance and $T_{C}$ is the Curie temperature. Equetion 1 is only valid above the Curie temperature [1,2]. 
Imagine a quadratic $2 \boldsymbol{d}$ area with $L^{2}$ spins on a grid. Each spin can either point up $(+1)$ or down $(-1)$. The average magnetization of the area is the average spin value and hence between 1 (completely ordered state) and 0. Neighboring spins $S$ and $S^{\prime}$ interact with an interaction energy of $E=-J S S^{\prime}$. Since each spin has 4 nearest neighbors (periodic boundary conditions), the interaction energy per spin can be between $(-4 J)$ (all neighbors parallel to the center spin) and $(+4 J)$ (all 4 neighbors antiparallel to center spin) where $(J)$ is the coupling strength. (There is no external magnetic field present) $[3,4]$.

Generally, states with less energy are preferred, so the system stays in completely ordered state for zero temperature. However, as we increase temperature, each spin has a thermal energy of $k_{B} T$ (where $k_{B}$ is Boltzmann's constant and $T$ is the absolute temperature). Due to this thermal energy, the system does not stay in completely ordered state but spins start to fluctuate ("flip") randomly.

There is a phase transition at the critical temperature of $\left(T_{C}=2.27 \mathrm{~J} / k_{B}\right)$ : Starting with a completely ordered state, the system stays mostly ordered below the critical temperature while it goes completely unordered above it.

Hence, above the critical temperature, the average magnetization is (about) zero while it is non-zero below it. On the two-dimensional lattice each spin interacts with its four neighbors as shown in Figure 1 [4].

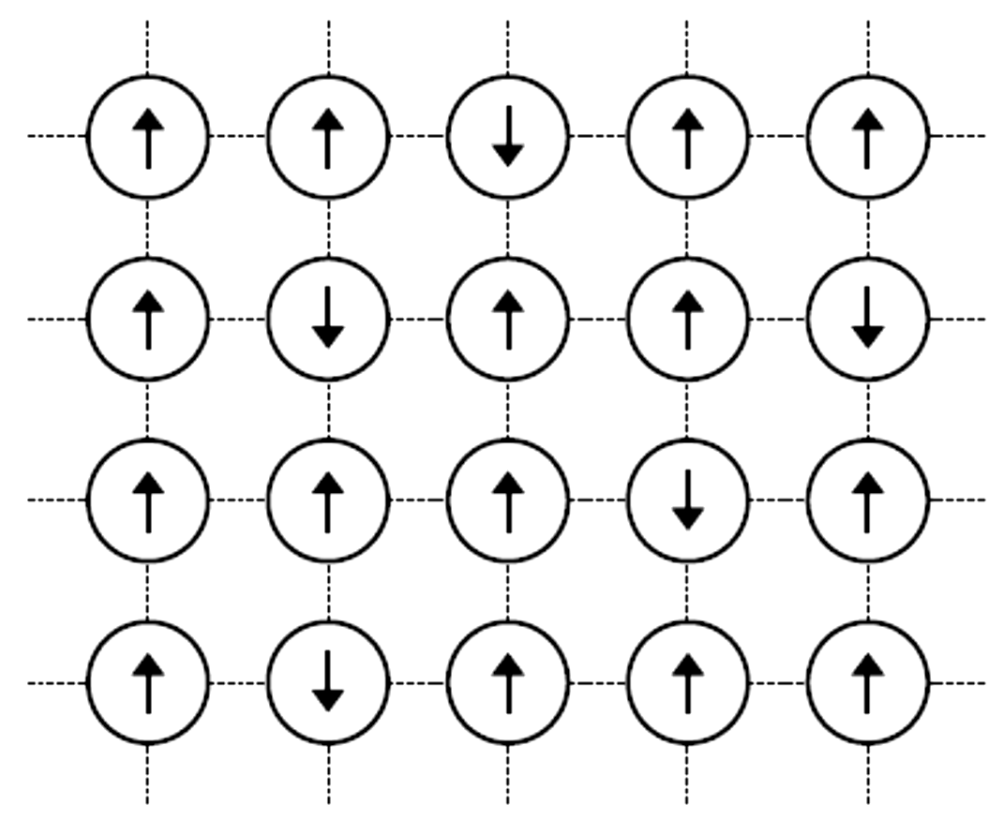

Figure 1. An example 2-dimensional Ising model spin configuration [5]. 


\section{THE ISING MODEL}

The Hamiltonian for a system that is dependent on the arrangement of spins on a lattice and from that we can deduce properties such as magnetization and susceptibility $[5,6,7]$. Suppose that the Hamiltonian is

$$
H=-J \sum_{\langle i j\rangle} s_{i} s_{j}-B \sum_{i} s_{i}
$$

$$
\text { internal interaction energy external magnetic energy }
$$

where $\langle i j\rangle$ means that we sum over the nearest-neighbor pair of spins. This means that the spin at site $i j$ interacts with spins at sites $i(j \pm 1)$ and $j(i \pm 1)$ respectively. We are assuming periodic boundary conditions in our model which means that every spin will interact with four other spins regardless of their position on the finite lattice. Here $J$ is the dimensionless interaction strength and $B$ represents the energy involved in the magnetization of the lattice and is also dimensionless.

The Ising model considers the problem in two dimensions and places dipole spins at regular lattice points while restricting their spin axis to be either up $(+y)$ or down $(-y)$.

The lattice configuration is square with dimensions $\boldsymbol{L}$ and the total number of spins equal to $N=L \times L$. In its simplest form the interaction range amongst the dipoles is restricted to immediately adjacent sites (nearest neighbors). This produces a Hamiltonian for a specific spin site, $\boldsymbol{i}$, of the form [8-10] :

$$
H_{i}=-J \sum_{j n n} s_{i} s_{j}
$$

where the sum $j_{n n}$ runs over the nearest neighbors of $i$. The coupling constant between nearest neighbors is represented by $J$ while the $S_{i}$ and $S_{j}$ are the respective nearest neighbor spins.

The nature of the interaction in the model is all contained in the sign of the interaction coupling constant $J$. If $J$ is positive it would mean that the material has a ferromagnetic nature (parallel alignment) while a negative sign would imply that the material is antiferromagnetic (favors anti-parallel alignment). $J$ will be taken to be +1 in our discussion and the values for spins will be +1 for spin up and -1 for spin down. A further simplification is made in that $J / k_{B}$ is taken to be unity.

The relative positioning of nearest neighbors of spins is shown in Figure 2 with the darker dot being interacted on by its surrounding neighbors $[9,10]$. 


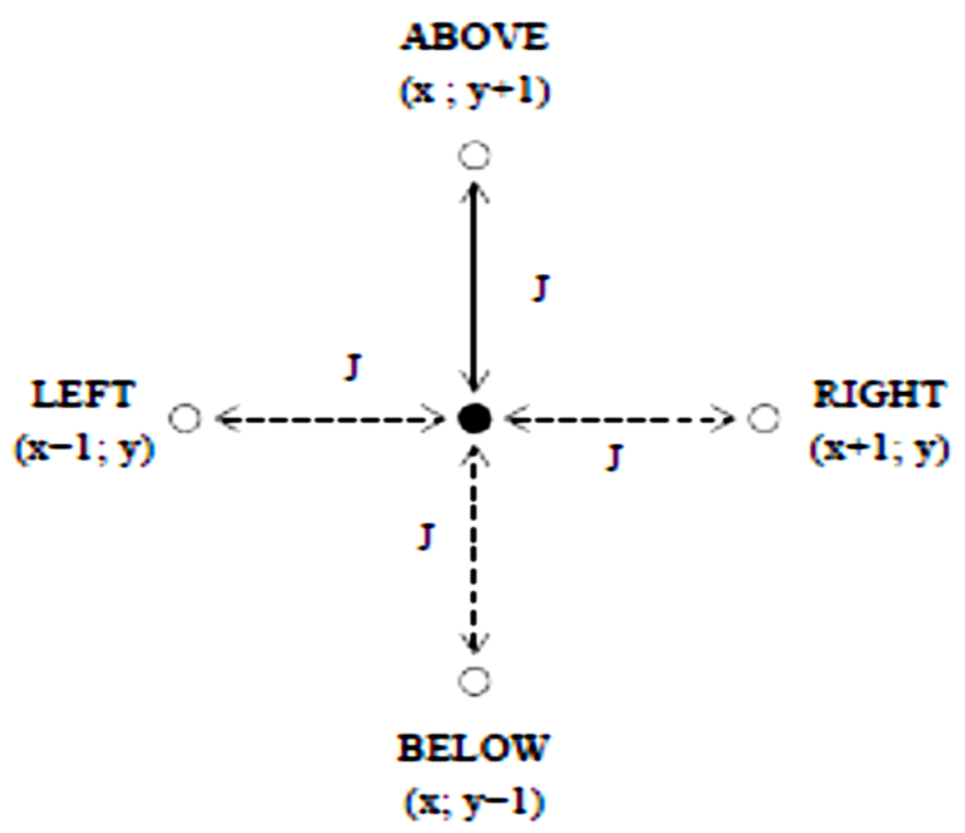

Figure 2. Nearest neighbor coupling. The dark dot, at position $(\mathrm{x}, \mathrm{y})$, is being interacted upon by its nearest neighbors which are one lattice spacing away from it [9].

In the simulation, whenever flipping a spin lowers the interaction energy, the flip is done. If it increases the energy, the flip is only committed with a probability of $\exp (-\beta E)$ where $\beta=1 / k_{B} T$ and $(E>0)$ is the energy difference between flipped and unflipped state (Metropolis algorithm). As one can see, the relevant temperature can be expressed in units of $\beta J$ which is called the reduced temperature and is the "natural" temperature unit used throughout the implementation [4].

The simulation repeatedly computes so-called MCS (Monte-Carlo step), commonly also referred to as time, each of whom involves the potential flipping (as explained above) of all spins in the area [4].

\section{RESULTS AND DISCUSSION}

\section{1. Effect of the size}

In order to see the effect of the size of the lattice on the transition of the phase, the square of the magnetization against $T$, for each size and for five temperatures in the range $\left(2.25-2.29 \mathrm{~J} / \mathrm{k}_{\mathrm{B}}\right)$ is plotted in Figure 3 for three lattice of sizes $(20,40,60)$ for $(200000$ steps each), in the absence of the external magnetic field.

In Figure 3, at low temperature below $\left(\mathrm{T}_{\mathrm{c}}=2.27 \mathrm{~J} / \mathrm{k}_{\mathrm{B}}\right)$ the square of the magnetization were all most stable for all lattice sizes.

While at higher temperatures above $\left(T_{c}=2.27 \mathrm{~J} / \mathrm{k}_{\mathrm{B}}\right)$ the square of the magnetization disorder and the fluctuation are larger for all lattice sizes . 


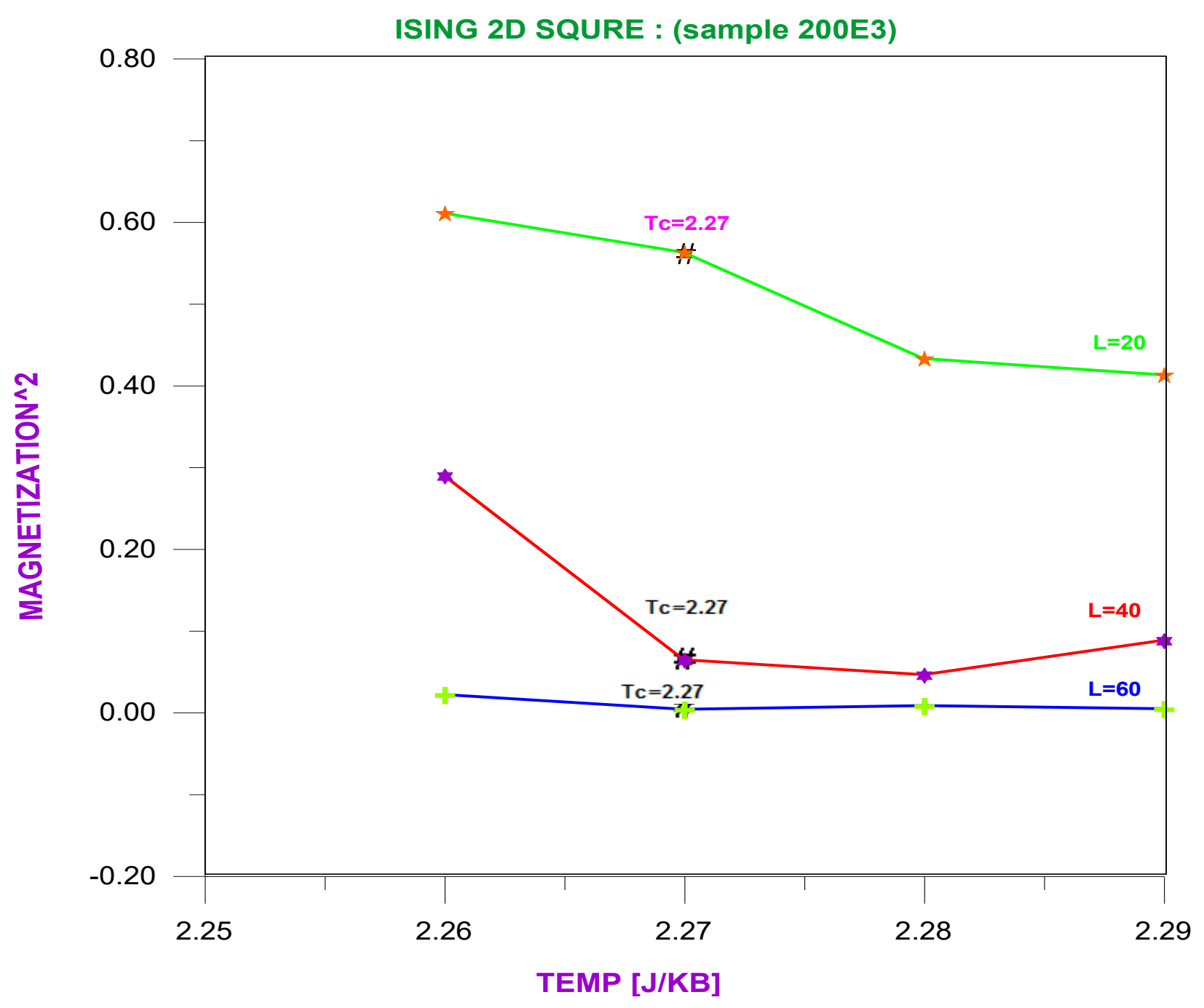

Figure 3. Magnetization square as a function of temperature at $\left(T_{C}=2.27 \mathrm{~J} / k_{B}\right)$ for three of kinds lattices $(20 \times 20,40 \times 40,60 \times 60)$, in the absence of the magnetic field .

\section{2. Relaxation}

Starting from a completely ordered state, this displays the average magnetization over time (i.e. MCS $)$ at a reduced temperature for lattice $(200 \times 200)$ in the absence of magnetic field $(B=0)$ has been shown in Figure 4.

In the first case (green graph) where the temperature is below the critical temperature the magnetization and the system stays mostly ordered, while in the second case (red graph) where the temperature is above the critical temperature the magnetization fluctuating thermically around zero and the system rapidly goes in to completely unordered state . 


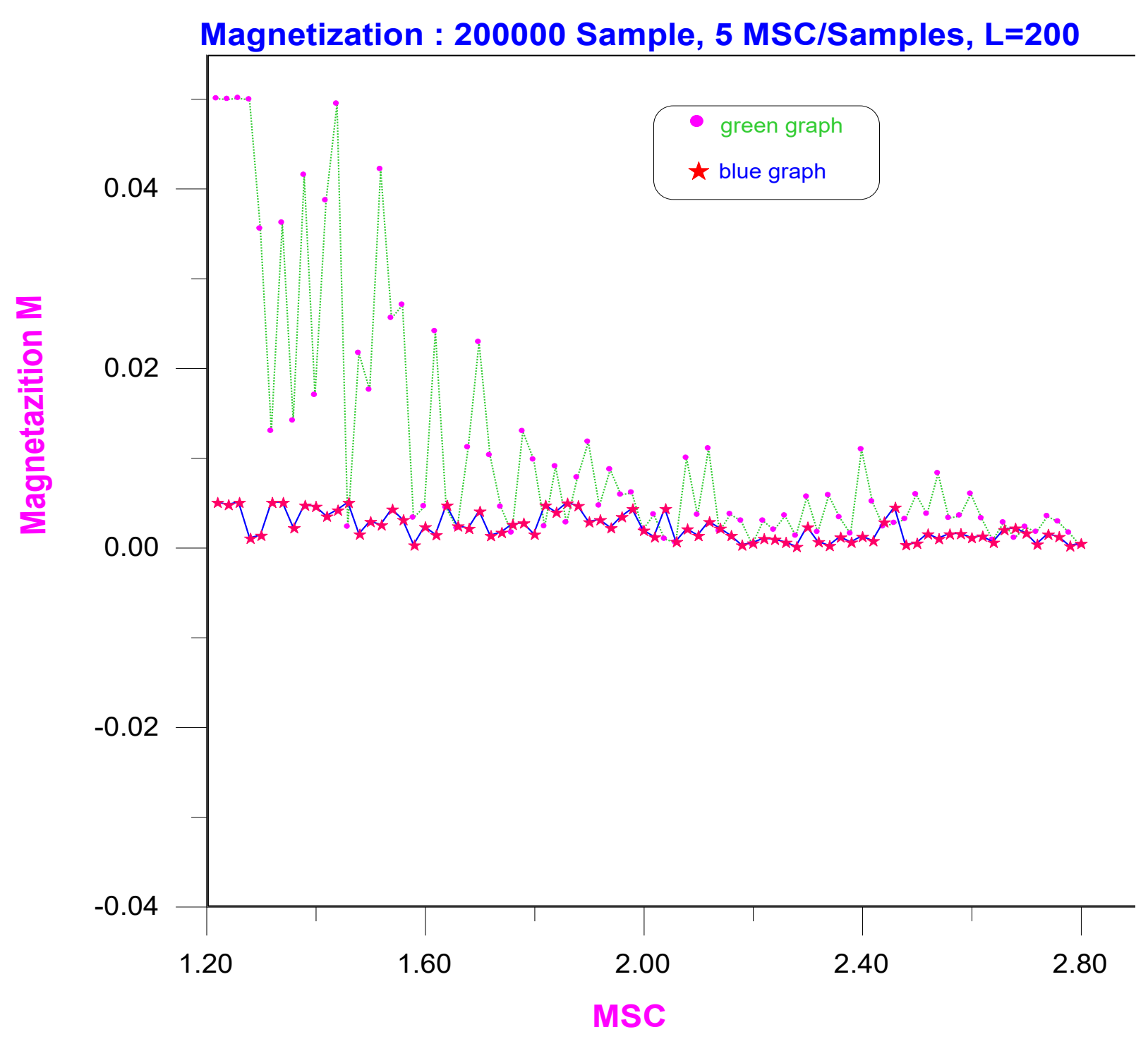

Figure 4. Average magnetization over time (i.e. MCS) at a reduced temperature for lattice $(200 \times 200)$ in the absence of the magnetic field.

\section{3. Magnetization}

The phase transition can most easily be seen when starting with a completely ordered state and computing the average magnetization after lots of (MCS) for different temperatures. Figure 5, shows the absolute value of the average magnetization versus temperature from ( 1 to $\left.4 J / k_{B}\right)$ for area sizes $(60 \times 60)$ (violet graph) and $(120 \times 120)($ blue graph $)$. For zero temperature, the state stays completely ordered for both lattice. As temperature increased, the magnetization of both lattice starts to drop more and more rapidly until reaching the phase transition (at critical temperature). Above the critical temperature $\left(T_{C}\right)$ of about $\left(J / k_{B}=2.27\right)$, the absolute average magnetization is nearly zero. The larger the area lattice $(120 \times 120)$ the 
more drastic the effect of the thermal fluctuations cancel better than the smaller area lattice $(60 \times 60)$ when averaging.

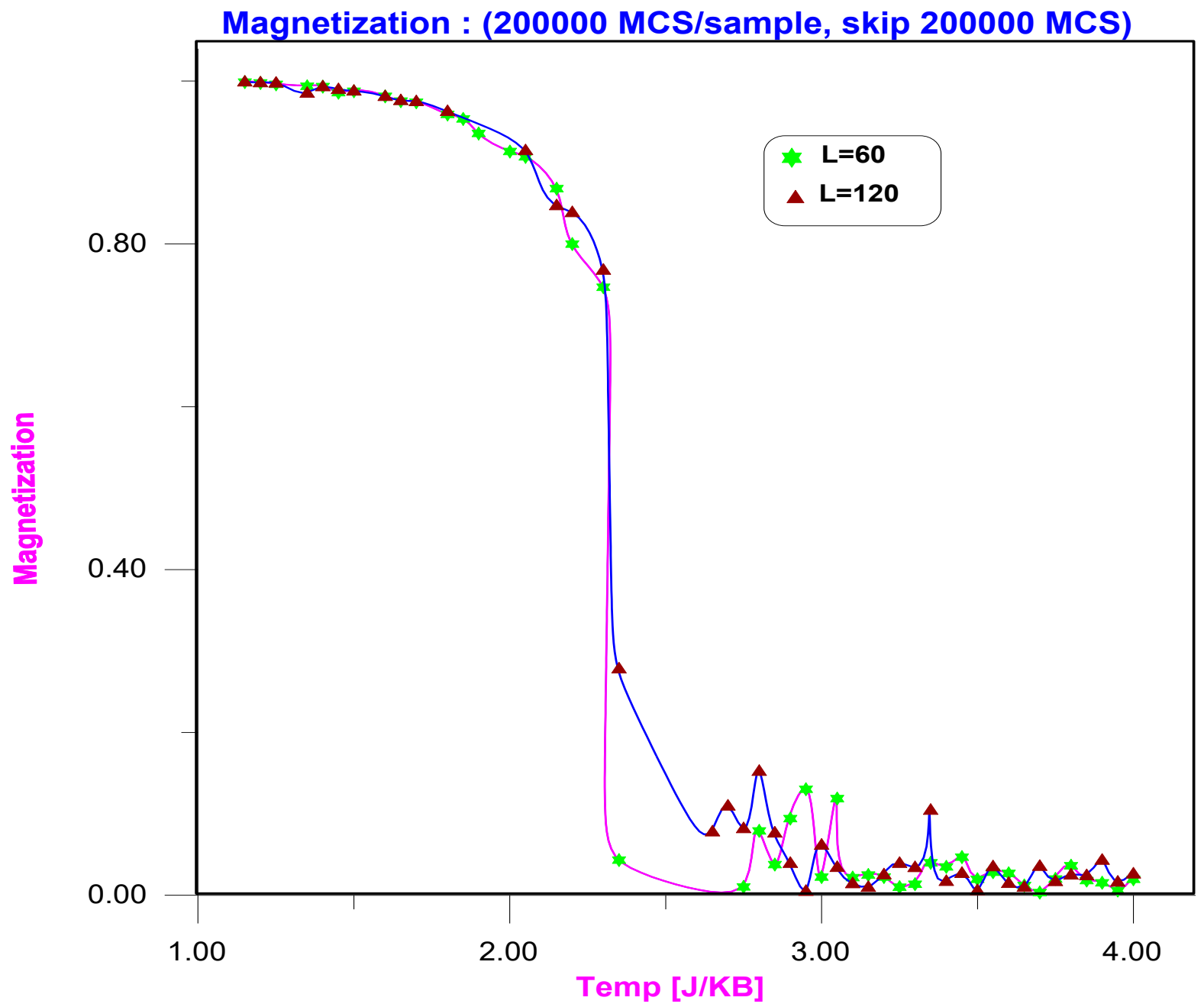

Figure 5. Magnetization as a function of temperature for two of kinds lattices $(60 \times 60,120 \times 120)$, in the absence of the magnetic field .

In Figure 6 a phase transition is clearly seen below $\left(T_{C}=2.27 \mathrm{~J} / k_{B}\right)$ for lattice $(20 \times 20)$ in the absence of the magnetic field.

At lower temperature, the system strongly favors the ground states. These are the states with all spins aligned, either all up $(M=1)$ or all down $(M=-1)$.

At temperature higher than the phase transition, the spins tend to be randomly aligned, which results in $(M=0)$.

This corresponds to a high temperature (the crossing of Curie's temperature) since $(J=-E / k T)$ which means that $(J)$ is inversely proportional to $T$. However, as the interaction strength increased the spins tend to align. 


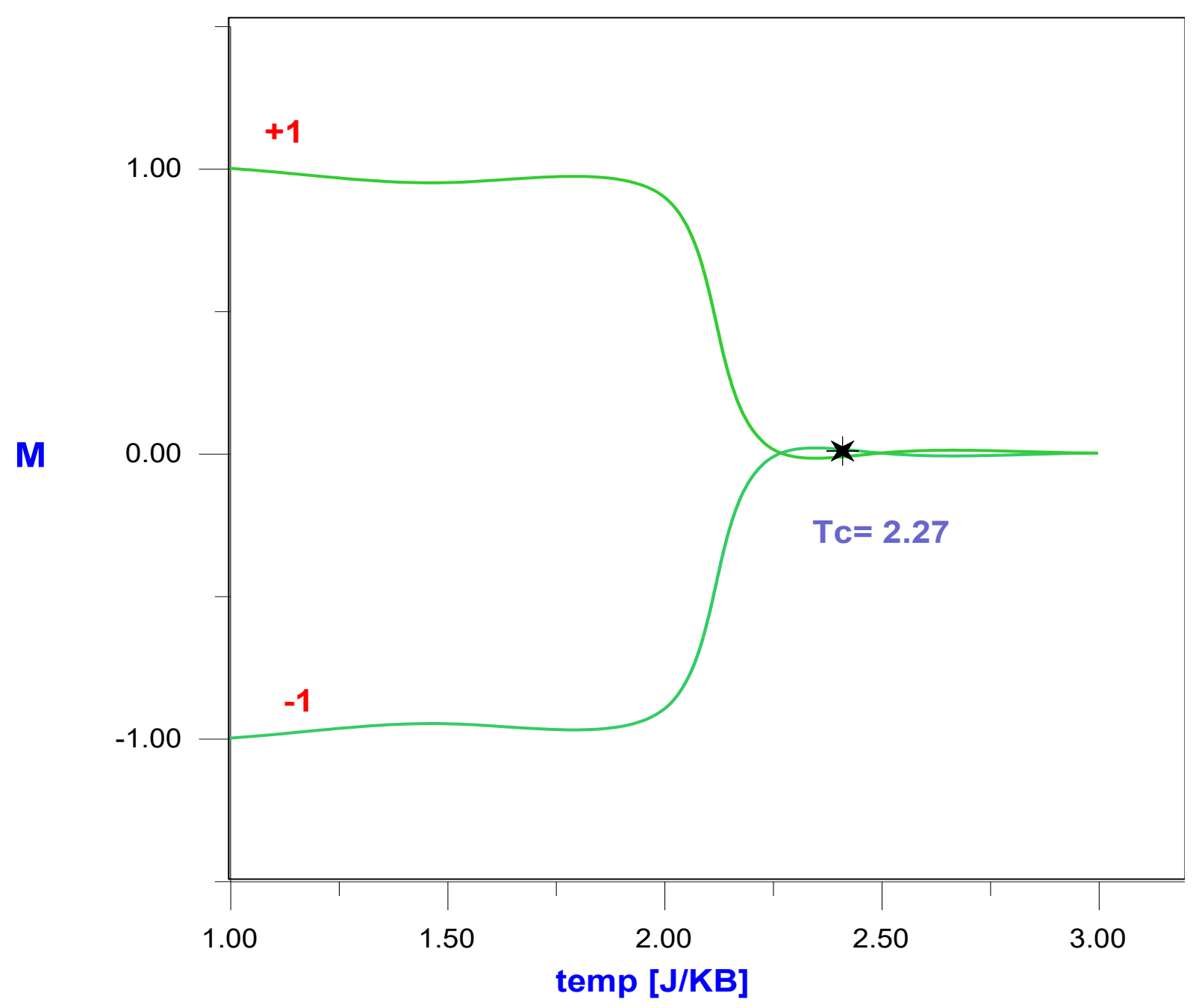

Figure 6. Magnetization as a function of temperature for the $(20 \times 20)$ particle lattice in the absence of the magnetic field.

\section{4. Susceptibility}

At $\left(\mathrm{T}>\mathrm{T}_{\mathrm{C}}\right)$ the susceptibility is in phase paramagnetic where the Curie-Weiss law $\left(X_{p}=\frac{C}{T-T_{C}}\right)$ can be applied from $\left(0-T_{C}\right)$ for $(40 \times 40)$ lattice (see Figure 7 ).

At high temperature $\left(\mathrm{T}>\mathrm{T}_{\mathrm{C}}\right.$ ) the paramagnetic susceptibility decrease and the effect of the thermal agitation appears to cause in neglecting the effect of the intrinsic molecular field. 
( Susceptibility X : For paramagnetization, L=40 ,Sample=100000)

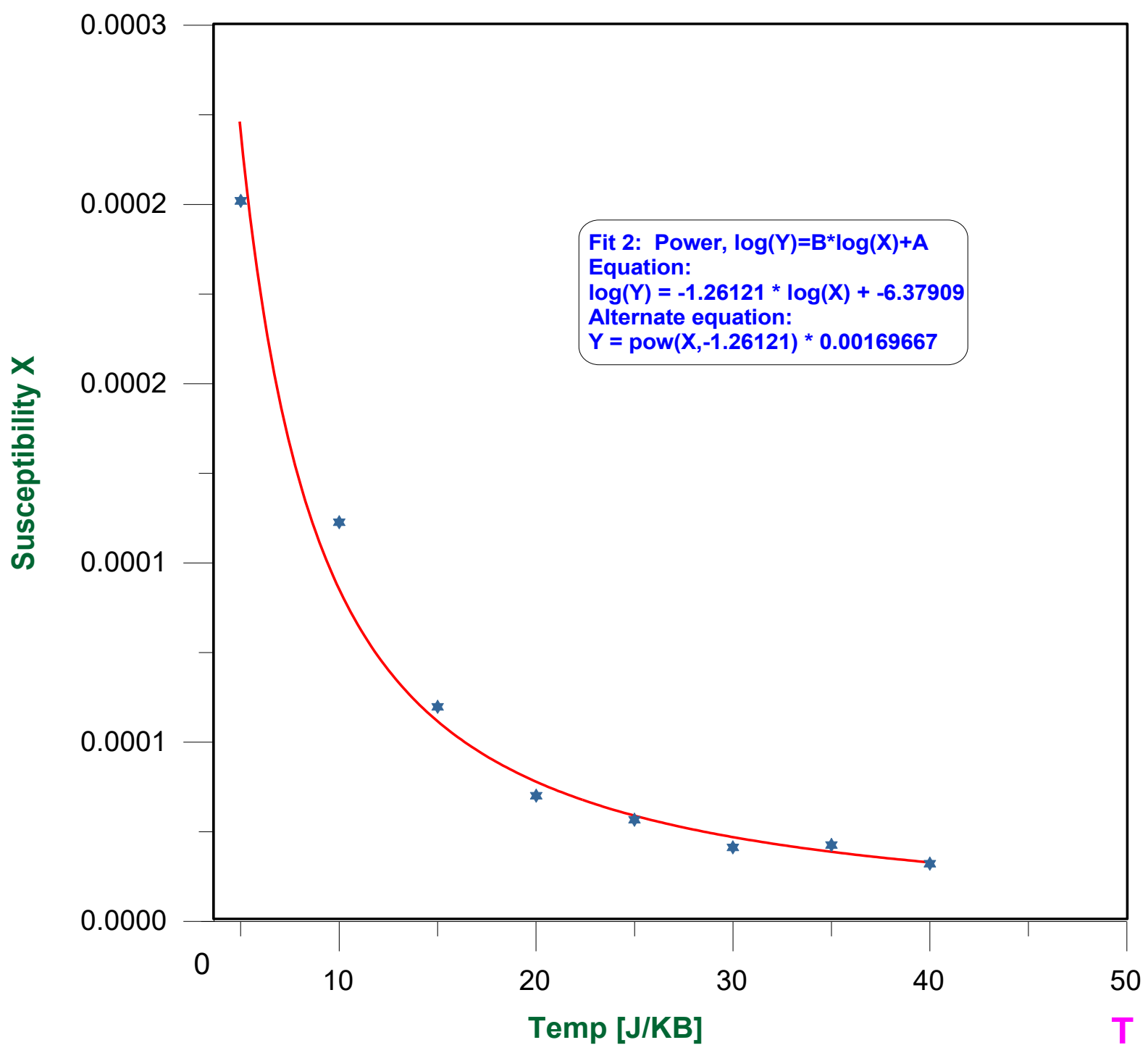

Figure 7. show the susceptibility paramagnetic region for lattice $(40 \times 40)$

The separation between the paramagnetic region and the ferromagnetic region for $(80 \times 80)$ lattice in the absence of the external magnetic field $(B=0)$ can be described in two ways . 
In Figure 8, where $\left(\mathrm{T}<\mathrm{T}_{\mathrm{C}}\right)$ the Curie-Weisslaw $\left(X_{p}=\frac{C}{T-T_{C}}\right)$ cannot be applied from $\left(0-T_{C}\right)$ as the susceptibility has negative values.

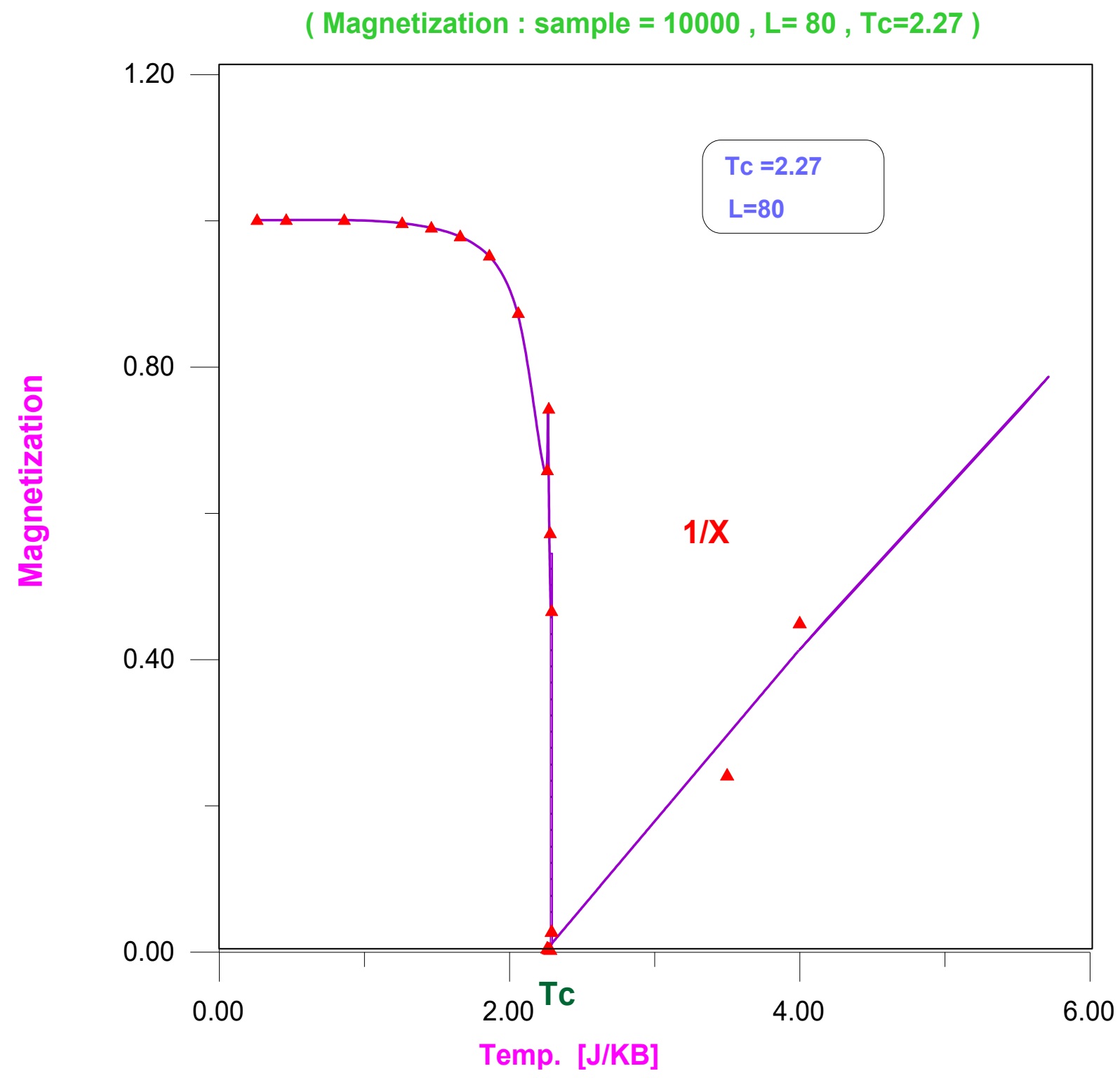

Figure 8. The paramagnetic region and the ferromagnetic region in lattice $(80 \times 80)$ 
This can be clear in Figure 9 where at $\left(T=T_{C}\right)$, the susceptibility $(x)$ goes to infinite value $(x \rightarrow \infty)$, while it becomes finite with high values until it becomes zero $(x=0)$.

This means that it has a spontaneous magnetization and becomes in the phase of the ferromagnetic state in the absence of magnetic field for lattice $(20 \times 20)$.

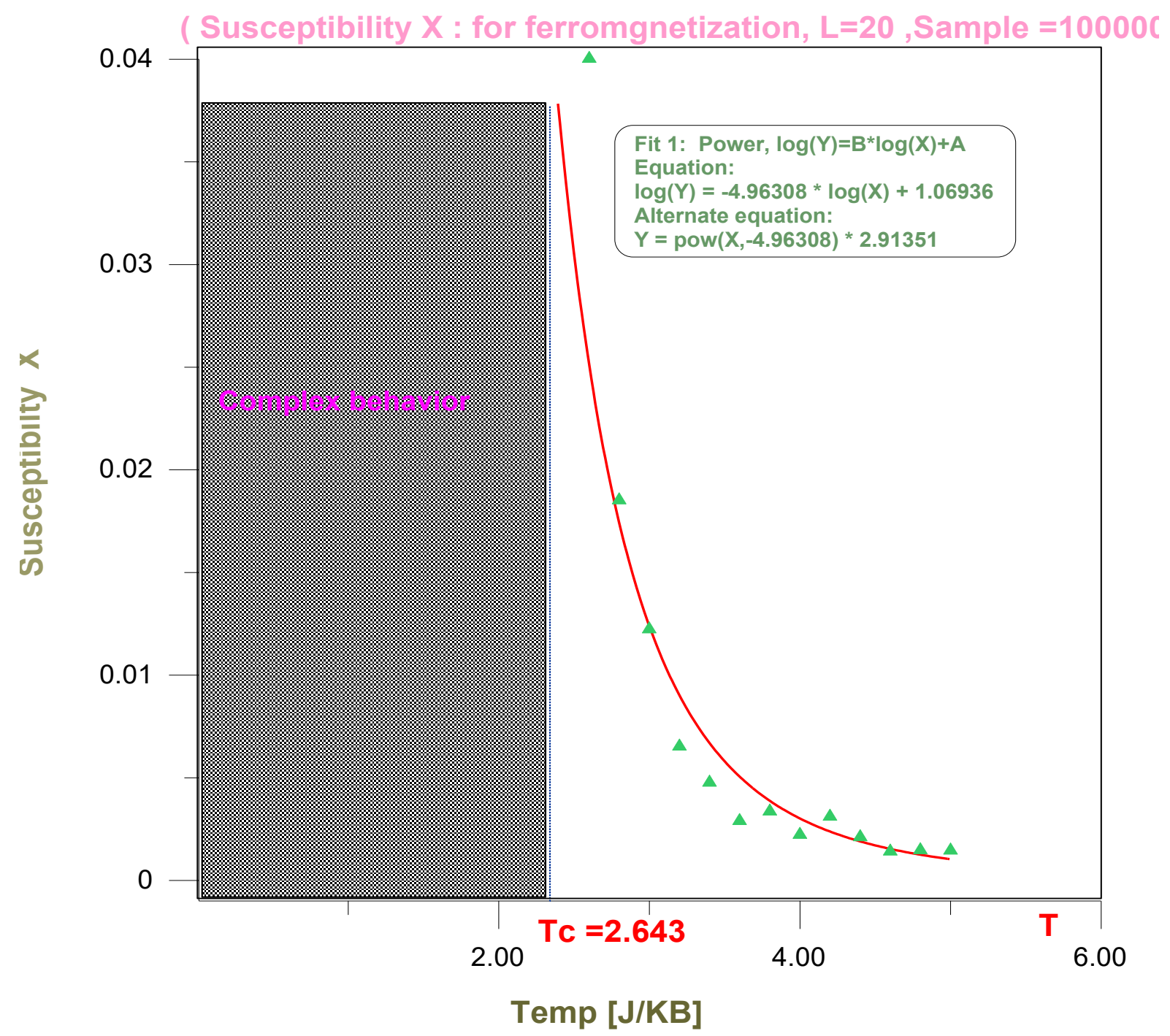

Figure 9. Susceptibility depends on temperature for ferromagnetic materials in lattice $(20 \times 20)$.

\section{CONCLUSION}

The Monte Carlo method applied to the Ising model which describes the magnetic properties of materials (lattices) allows to obtain the thermodynamic quantities variations with 
different lattice sizes at Curie temperature $\left(T_{C}=2.27 \mathrm{~J} / k_{B}\right)$. Above a certain temperature (T $>\mathrm{T}_{\mathrm{C}}$ ) and in the absence of the magnetic field $(B)$, the spins are randomly oriented, a phase transition will be paramagnetic state, leading to decreasing the susceptibility and magnetization and it has no effect because of the thermal agitation. In a certain temperature at $\left(\mathrm{T}=\mathrm{T}_{\mathrm{C}}\right)$ where there will be a phase transition, the magnetization decreases causing the magnetic susceptibility to goes to infinity. At $\left(T<T_{C}\right)$ the spins are aligned, hence the average magnetization will increased and the phase transition will be in a ferromagnetic state.

\section{References}

[1] Madison, Wisconsin 53706, Ferromagnetism-The Curie Temperature of Gadolinium, Advanced Laboratory, Physics 407,University of Wisconsin, (4/9/2003).

[2] Charles Kittel, Introduction to Solid State, Inc. See the sections on Paramagnetism and Ferromagnetism, Physics 6th ed.(1986) John Wiley \& Sons.

[3] Robert Knegjens, Simulation of the 2D Ising Model, May 13, 2008 .

[4] Wolfgang Wieser, Simple ising model magnetization simulation, Copyright (C) 2004-2008 by Last modified: 2008-01-22 02:49:17 .

[5] Indrek Mandre, The ising model, Course project in simulation of physical processes, Tallinn University of Technology, Dept. of Physics, Tallinn 2008 .

[6] Jon Emil Gudmundsson, Monte Carlo method and the Ising model, University of Uppsala, Course: Statistical methods in Physics. Teacher: Gunnar Ingelman, 2010 .

[7] Stefan Sellner, Ising model Calculations using the Monte-Carlo method, March 11, 2008.

[8] Tobin Fricke, Monte Carlo investigation of the Ising model, Taken on the $9^{\text {th }}$ of March 2008, March 11, 2008.

[9] Jacques Kotze, Introduction to Monte Carlo methods for an Ising Model of a Ferromagnet, 3Mar 2008.

[10] Matthias Reggentin, Monte Carlo Methods in Physics Ising model and Metropolis Algorithm, reggentin@physik.hu-berlin.de, 01-16-2010. 